\title{
Free Boundary Conditions at Austenite-Martensite Interfaces
}

\author{
O. P. Bruno \\ School of Mathematics, Georgia Institute of Technology, Atlanta, GA 30332-0160 \\ P. H. Leo \\ Dept. of Aerospace Engineering and Mechanics, Univ. of Minnesota, Minneapolis, MN 55455 \\ F. Reitich \\ Center for Research in Scientific Computation and Dept. of Mathematics, North Carolina State \\ University, Raleigh, NC 27695-8205 \\ (January 24, 1995)
}

\begin{abstract}
A mathematical model is presented for the dynamical strain-stress response of shape memory wires. This model is based on a free boundary problem for the heat equation which incorporates bistable potentials together with a physical constant, independent of stress or temperature, which accounts for mechanically dissipative mechanisms. An expression in closed form is derived for one of the most important observables, namely the width of the hysteresis loops as a function of the elongation rates. Excellent agreement with experimental results is observed.
\end{abstract}

PACS numbers: $64.70 . \mathrm{Kb}, 65.50 .+\mathrm{m}, 68.48 .+\mathrm{f}, 82.65 . \mathrm{Dp}$

Typeset using REVTEX 
Shape memory alloys (SMA) and the pseudoelastic hysteresis they exhibit have received sustained attention in recent years [1-11]. The interest in these alloys, which undergo a structural phase transition from a high temperature phase (austenite) to a low temperature phase (martensite), stems in part from their applicability as elements in active structures. The shape change which accompanies the phase transition is characterized by a large misfit strain $\epsilon^{T}(\approx 10 \%)$ in the martensitic phase; some materials, primarily NiTi, can undergo this transition thousands of times without showing noticeable changes in their behavior.

A recent set of experimental results on NiTi shape memory wires [8] prompted a number of theoretical investigations $[8,12]$. These experimental results showed that the widths of the stress-strain hysteresis associated with these wires depend on the corresponding elongation rates; previous observations of similar phenomena were reported in [10]. The dependence of the hysteresis widths on the strain rates was attributed in [8] to an interplay between the thermodynamics of the phase transition and heat flow through conduction and convection. A one-dimensional discrete model presented in that paper shows quantitative agreement with the experimental results. That model is, in fact, the first model in the literature showing quantitative agreement with observed dynamical behavior of SMA's.

In what follows we present a related continuous free-boundary model, and we use its asymptotics to derive a closed-form expression which relates the most important experimental observables. This formula provides new insights on the physical phenomena under consideration. In particular, it predicts correctly the experimental observation of a critical value of the stress which may not be exceeded before the wire has transformed completely. The continuous formulation allows, in addition, for application of sophisticated free boundary solvers to our phase transition problem, and thus permits us to obtain very accurate numerical solutions. Comparison of numerical results with our explicit expression yields good agreement for a wide variety of configurations. Departure from such agreement, which is observed in some extreme regimes, shows that in such cases transient heat transfer effects are responsible, in part, for the behavior of the wire. As argued in [12], our approach is in marked contrast with others in which the velocity of the free boundary is prescribed 
by certain functions of stress and temperature, and which only allow for predictions of a qualitative nature, see e.g. [1].

The quasi-static discrete model [8] is based on consideration of the energetics of shape memory alloys, the release of latent heat and shape change associated with the phase transition, and the conduction, convection and dissipation of heat in the wire and its surroundings. According to this model, an overall elongation $\Delta L$ imposed in a time interval $\Delta t$ can be accommodated either by means of small elastic deformations, by transformation of austenite into martensite (occurring through propagation of an austenite-martensite interface) or by a combination thereof. Small elastic deformations account for the incremental strain if the stress in the wire is below the critical stress $\bar{\sigma}^{\text {load }}=\sigma_{M}\left(\theta_{\text {int }}\right)+\sigma^{\text {hyst }}$ on loading, or above $\bar{\sigma}^{\text {unload }}=\sigma_{M}\left(\theta_{\text {int }}\right)-\sigma^{\text {hyst }}$ on unloading, at which the phase transition from austenite to martensite is possible. Otherwise, elongations which include phase transformation must occur, at a stress equal to the (discretized value of the) critical stress. Here $\theta_{\text {int }}$ is the interface temperature, and $\sigma_{M}=\sigma_{M}(\theta)$ is the Maxwell stress associated with the temperature $\theta$, see equation (5) below. The stress $\sigma^{\text {hyst }}$ is a material constant that accounts for dissipative forces in the wire $[8,12]$.

It follows that in the limit of small $\Delta L$ the interface moves with a continuous velocity, and the stress in the wire must equal the (temperature dependent) transition stress $\bar{\sigma}$ as long as the interface propagates. This is an important observation, since it allows us to model our physical configuration by means of a continuous free boundary problem for the heat equation. Let us call $\theta=\theta(x, t)$ the temperature in the wire, $\theta_{0}$ the ambient temperature, $l$ the latent heat of transformation per unit volume and set $l^{*}=l+\sigma^{h y s t} \epsilon^{T}$. Further call $s=s(t)$ the reference position of the interface at time $t$ (i.e. the position of the interface as measured in the reference unstrained configuration). Then neglecting small heat transfer effects due to elongations and assuming insulated attachments at the ends of the wire, the foregoing considerations imply the following set of equations for the temperature profile $\theta(x, t)$ and the interface $s=s(t)$ in a wire of length $L_{0}$ : 


$$
\begin{gathered}
\rho c_{p} \theta_{t}=k \theta_{x x}-\frac{2 h}{r}\left(\theta-\theta_{0}\right), \quad 0<x<\frac{L_{0}}{2}, \\
{\left[k \theta_{x}\right]_{-}^{+}(s(t), t)=-l^{*} \dot{s}(t)}
\end{gathered}
$$

and

$$
\left\{\begin{array}{lr}
\sigma=\left(\sigma_{M}(\theta(s(t), t)) \pm \sigma^{h y s t}\right) & \text { for } \pm \dot{s}>0 \\
\left|\sigma-\sigma_{M}(\theta(s(t), t))\right| \leq \sigma^{h y s t} & \text { for } \dot{s}=0
\end{array}\right.
$$

with initial and boundary conditions given by

$$
\theta(x, 0)=\theta_{0}, \quad s(0)=0 \text { and } \theta_{x}(0, t)=\theta_{x}\left(\frac{L_{0}}{2}, t\right)=0
$$

In equations (1) and (2), $\rho$ is the mass density of the unstrained wire, $c_{p}$ its specific heat, and $k, h$ and $r$ its thermal conductivity, coefficient of convection of heat and radius, respectively. Equations (1)-(4) reflect the experimental observation in [8] of two interfaces, one starting from each end (the action of the grips thus appearing as an agent which favors nucleation). Indeed, symmetry considerations on a wire of length $L_{0}$ show that we must have $\theta_{x}\left(\frac{L_{0}}{2}, t\right)=0$ at all times.

Provided a constitutive relation for the Maxwell stress $\sigma_{M}$ such as (8) below is known, and that the stress $\sigma$ in equation (3) is prescribed in accordance with a given experimental configuration (see e.g. equation (7) below), equations (1)-(4) constitute a well defined free boundary problem for the heat equation. Free boundary problems which, like ours, contain free boundary conditions allowing for a range of values of the unknown temperature for $\dot{s}=0$ are known in the theory of solidification by the name of "Stefan-Signorini problems". (See [13] for an existence and uniqueness theory for some instances of such problems). Interestingly, in some cases our mathematical model coincides with those describing certain liquid-solid phase transitions. Indeed, the free boundary problem associated with a stress controlled configuration can easily be translated into a set of equations which is identical to that associated with a two-phase solidification problem where the liquid is initially supercooled, see e.g. [14-17]. As is known, such equations can lead to predictions of infinite 
interface velocities. This is in agreement with our equation (6) below, which predicts infinite speeds for certain values of the imposed stress.

The main new element in our equations, namely the free boundary condition (3), can be obtained from the principle of conservation of energy under the following three assumptions: 1) The constant stress $\sigma^{\text {hyst }}$ provides the only dissipative mechanism and the dissipation occurs at the interface (the complementary conservative stress $\sigma^{\text {cons }}$ is therefore given by $\sigma_{I}^{\text {cons }}=\sigma-\sigma^{\text {hyst }}$ at the interface, and by $\sigma_{0}^{\text {cons }}=\sigma$ away from the interface), 2) Strain and energy differences arising from small elastic deformations caused by the stress $\sigma^{\text {hyst }}$ in a transforming mass element can be neglected as much smaller than corresponding differences associated with the phase change of the element, and 3) Temperature changes in a mass element are sufficiently small that increments $\psi \Delta \theta$ can be neglected as much smaller than the corresponding changes in the (bistable) specific Helmholtz free energy $h$ due to the incremental strains: $\rho \Delta h=-\rho \psi \Delta \theta+\sigma^{\text {cons }} \Delta \epsilon \approx \sigma^{\text {cons }} \Delta \epsilon$. (Here we have denoted by $\psi$ the specific entropy and by $\Delta \theta$ and $\Delta \epsilon$ the temperature and strain changes, respectively, which occur at a given point in the wire during a time interval $\Delta t$ ). Notice that in the limit of very slow elongations the process is truly isothermal and we have $\psi \Delta \theta=0$.

The conditions (3) follow directly from these assumptions. To obtain the first one of these, for example, we note that assumption 2) implies the relations $\Delta h \approx\left(h\left(\epsilon^{m}, \theta\right)-h\left(\epsilon^{a}, \theta\right)\right)$ and $\Delta \epsilon \approx \epsilon^{T}$ for the energy and strain change in an element which undergoes the phase transition. Here $\epsilon^{m}$ and $\epsilon^{a}$ are the strains at which the martensite and austenite phases can coexist in an equilibrium configuration, and $\epsilon^{T}=\epsilon^{m}-\epsilon^{a}$. (Note that in our dynamic configuration and due to the additional dissipative stress, the actual strains in the austenite and martensite phases are different from the corresponding static ones). Assumptions 1) and 3) then give us $\rho \Delta h \approx \sigma_{I}^{\text {cons }} \epsilon^{T}$ and thus $\sigma_{I}^{\text {cons }} \approx \sigma_{M}$ where $\sigma_{M}$, the Maxwell stress at temperature $\theta$, is given by

$$
\sigma_{M}=\frac{\rho\left(h\left(\epsilon^{m}, \theta\right)-h\left(\epsilon^{a}, \theta\right)\right)}{\epsilon^{m}-\epsilon^{a}}
$$

as desired. 
The free boundary problem (1)-(4) can be used to derive simple expressions in closed form giving observables such as the width of the hysteresis loops and temperature changes as function of the experimental parameters. To find these expressions we propose a solution $\{\theta(x, t), s(t)\}$ in the form of a traveling wave in an infinite wire. Let the traveling temperature profile be given by $u=u(x)=\theta(x+v t, t)-\theta_{0}$ and $s(t)=v t$. Equation (1) now translates into an ordinary differential equation for the unknown $u$ whose solution is

$$
u(x)= \begin{cases}\delta \theta e^{\lambda^{-} x} & \text { for } x>0 \\ \delta \theta e^{\lambda^{+} x} & \text { for } x<0\end{cases}
$$

where $\lambda^{ \pm}=\frac{1}{2}\left(-\frac{\rho c_{p v}}{k} \pm \sqrt{\left(\frac{\rho c_{p v}}{k}\right)^{2}+\frac{8 h}{r k}}\right)$ and $\delta \theta=\left(\theta_{i n t}-\theta_{0}\right)$. Condition (2) on the jump of the derivative across the interface then becomes a relation between the temperature increase and the speed of the interface

$$
l^{*} v=\delta \theta \sqrt{\left(\rho c_{p} v\right)^{2}+8 h k / r}
$$

Let us apply this expression to a strain-controlled experiment, on loading, and with an imposed strain rate $\dot{\epsilon}$. The stress $\sigma$ in (3) equals

$$
\sigma=\sigma(s(t), t)=C \frac{\left(\dot{\epsilon} t L_{0}-2 \epsilon^{T} s(t)\right)}{L_{0}}
$$

during transformation, where $C$ denotes the elastic modulus. From (7) we see that the asymptotic interface velocity is $v=\dot{\epsilon} L_{0} / 2 \epsilon^{T}$ and, using (3) and (6), the asymptotic temperature $\theta^{\text {asym }}$ and corresponding stress $\sigma^{\text {asym }}$ can be found. In a stress-controlled experiment, on the other hand, the value of $\sigma$ is imposed, e.g. $\sigma=\sigma(t)=\sigma^{*} \quad\left(\sigma^{*}=\right.$ const. $\left.>0\right)$. In view of equation (3), this prescription implies a value for the temperature which, together with equation (6) gives us the asymptotic values of the velocity of the interface and thus the associated elongation rate. These asymptotic calculations provide predictions which are in close agreement with numerical solutions and experimental results for a wide range of experimental parameters.

In Tables 1 and 2 we compare results given by equation (6) with corresponding numerical values obtained by integration of the system (1)-(4) by the method of lines $[18,19]$. In the 
strain-controlled case (Table 1), we also show experimental results as reported in [8]. For the small range of temperatures considered here it is reasonable to assume, as in [8], a linear dependence of the Maxwell stress on temperature

$$
\sigma_{M}(\theta)=\sigma_{0}+\eta\left(\theta-\theta_{0}\right)
$$

For the experiment under consideration we have $\theta_{0}=300^{\circ} \mathrm{K}, \sigma_{0}+\sigma^{\text {hyst }}=350 \mathrm{MPa}, L_{0}=$ $13 \mathrm{~cm}$ and $\eta=7.6 \mathrm{MPa} /{ }^{\circ} \mathrm{K}$. With the exception of $h^{\text {water }}$, the values of all physical constants we use coincide with those of [8]. Those values were either taken from the recent literature as cited in [8], or, in the case of $\sigma_{0}$ and $\sigma^{\text {hyst }}$, determined from the simple experiments indicated in that paper. Following [8], and due in part to the uncertainty in the values obtained for the latent heat of transformation, we have neglected the small amount of heat introduced by the dissipative stress $\sigma^{\text {hyst }}$, and we have taken $l^{*}=l=43 \mathrm{~J} / \mathrm{cm}^{3}$. The figure $h^{\text {water }}=0.089 \mathrm{~W} / \mathrm{cm}^{2}-^{\circ} \mathrm{K}$ used in [8], on the other hand, is an engineering value for the convection coefficient corresponding to a cylinder submerged in an infinite bath of stationary water. Since a flow of a thin film of water occurred in the experimental setup of [8], those authors used this value of $h^{\text {water }}$ together with a modified value of the specific heat which would account for configurational differences. It appears now that this procedure is not well justified, that the convection coefficient must be one which accounts for the actual water flow, and that the true value of the specific heat of the wire must be used, as we do below. Rough estimates based on consideration of convection rates for water moving on a heated plate together with comparison of theoretical predictions and experimental results lead us to consider the much larger value $h^{\text {water }}=0.4 \mathrm{~W} / \mathrm{cm}^{2}-^{\circ} \mathrm{K}$ used below. The fact that close agreement was obtained between theoretical and experimental results for all three values of the strain rate considered confirms the plausibility of this value of $h^{\text {water }}$, whose accurate determination will be left for future work. Since equation (6) is only exact in the limit of an infinitely long wire, we include, for comparison, numerical results corresponding to a wire with $L_{0}=13$ as well as those given for a much longer specimen $\left(L_{0}=6 \times 13 \mathrm{~cm}\right)$; in most cases the differences in predictions associated with the various lenghts are negligible. 
In Figures 1 and 2 we show strain-stress curves and the temperature profiles predicted by our theory. These curves were obtained by numerical integration of equations (1)-(4) by the method of lines [18]; comparison with the experimental curves of [8] again shows good agreement. The temperature profiles shown on the right side of the Figures are snapshots taken at the time for which half of the wire had transformed into martensite.

\section{ACKNOWLEDGMENTS}

We thank G. Meyer for valuable discussions and for providing a method-of-lines code. Stimulating conversations with M. Gurtin are also acknowledged. OB gratefully acknowledges support from NSF through grant No. DMS-9200002 and through an NYI award, and from the Sloan Foundation through the fellowship program. 


\section{REFERENCES}

[1] R. Abeyaratne and J. K. Knowles, J. Mech. Phys. Solids 41, 541 (1993).

[2] J. M. Ball and R. D. James, Arch. Rational Mech. Analysis 10013 (1987).

[3] K. Bhattacharya and R.V. Kohn, in Proceedings of the International Society for Optical Engineering 1919, Mathematics in Smart Structures (1993), p. 207.

[4] J. L. Ericksen, Journal of Elasticity 5, 191 (1975).

[5] J. D. Eshelby, Proc. Roy. Soc. London A 241, 376 (1957).

[6] F. Falk, Acta Metall. 28, 1773 (1980).

[7] R. James and D. Kinderlehrer, in Partial differential equations and continuum models of phase transitions, edited by M. Rascle, D. Serre and M. Slemrod, Lecture Notes in Physics Vol. 344 (1989), p. 51.

[8] P. H. Leo, T. W. Shield and O. P. Bruno, Acta Metall. 41, 2477 (1993).

[9] F. C. Lovey, A. Amengual, V. Torra and M. Ahlers, Phil. Mag. A 61, 159 (1990).

[10] Mukherjee, K., Sircar, S., and Dahotre, N., Material Science and Engineering, 41, 75 $(1985)$

[11] I. Müller and H. Xu, Acta Metall. 39, 263 (1991).

[12] O. P. Bruno, To appear in Smart Materials and Structures.

[13] A. Friedman and L. S. Jiang, J. Diff. Equations 51, 213 (1984).

[14] A. Fasano, M. Primicerio and A. Lacey, Quart. Appl. Math. 38, 439 (1981).

[15] I. G. Götz and B. Zaltzman, Preprint (1993).

[16] M. E. Gurtin, Quart. Appl. Math. 52, 133 (1994).

[17] B. Sherman, SIAM J. Appl. Math. 20, 555 (1971). 
[18] G. Meyer, SIAM J. Numer. Anal. 10, 522 (1973).

[19] J. Crank, in Methods in heat transfer, edited by R. W. Lewis, K. Morgan and O. C. Zienkiewicz, (John Wiley \& Sons, Chichester 1981), p. 177. 